University of Nebraska - Lincoln

DigitalCommons@University of Nebraska - Lincoln

May 2001

\title{
A LANDAU-GINZBURG DESCRIPTION OF Sb OVERLAYERS
}

\author{
Ralph Skomski \\ University of Nebraska-Lincoln, rskomski2@unl.edu \\ T. Komesu \\ University of Nebraska-Lincoln, tkomesu2@unl.edu \\ H.-K. Jeong \\ University of Nebraska-Lincoln, hjeong@unl.edu \\ C.N. Borca \\ University of Nebraska-Lincoln \\ Peter A. Dowben \\ University of Nebraska-Lincoln, pdowben@unl.edu \\ See next page for additional authors
}

Follow this and additional works at: https://digitalcommons.unl.edu/physicsdowben

Part of the Physics Commons

Skomski, Ralph; Komesu, T.; Jeong, H.-K.; Borca, C.N.; Dowben, Peter A.; Ristoiu, D.; and Nozieres, J.P., "A LANDAU-GINZBURG DESCRIPTION OF Sb OVERLAYERS" (2001). Peter Dowben Publications. 169.

https://digitalcommons.unl.edu/physicsdowben/169

This Article is brought to you for free and open access by the Research Papers in Physics and Astronomy at DigitalCommons@University of Nebraska - Lincoln. It has been accepted for inclusion in Peter Dowben Publications by an authorized administrator of DigitalCommons@University of Nebraska - Lincoln. 


\section{Authors}

Ralph Skomski, T. Komesu, H.-K. Jeong, C.N. Borca, Peter A. Dowben, D. Ristoiu, and J.P. Nozieres 


\title{
A LANDAU-GINZBURG DESCRIPTION OF Sb OVERLAYERS
}

\author{
R. SKOMSKI, ${ }^{1}$ T. KOMESU, ${ }^{1}$ H.-K. JEONG,${ }^{1}$ C. N. BORCA, ${ }^{1}$ P. A. DOWBEN, ${ }^{1}$ D. \\ RISTOIU ${ }^{2}$ and J. P. NOZIERES ${ }^{2}$ \\ ${ }^{1}$ Department of Physics and Astronomy and Center for Materials Research and Analysis, \\ University of Nebraska, Lincoln, NE 68508. \\ ${ }^{2}$ CNRS, Laboratoire Louis Néel, Avenue des Martyrs BP 166, 38042 Grenoble, \\ CEDEX 09, France.
}

\begin{abstract}
The spin polarization of $\mathrm{Sb}$ overlayers on the semi-Heusler alloy NiMnSb is investigated in terms of the Landau-Ginzburg approach. The half-metallic semi-Heusler alloy $\mathrm{NiMnSb}$ acts as a ferromagnetic perturbation and induces a spin polarization in the semimetallic Sb overlayer. Using a Gaussian approximation, the propagation of the spin perturbation in the overlayer is calculated. The results are compared with spin-polarized inverse photoemission spectroscopy (SPIPES) results and with recent spin-dependent envelope-function approximation (SDEFA) predictions. The Landau-Ginzburg parameters are both band-structure and temperature dependent, and it is argued that thermal spin excitations lead to an injection depth decreasing as 1//T law at high temperatures.
\end{abstract}

\section{INTRODUCTION}

The spin structure at interfaces is key to understanding spin electronics. Of particular interest are interfaces between different classes of materials, such as interfaces between magnetically ordered and semiconducting materials. Here we focus on the interface between a halfmetallic high-polarization ferromagnet and a semimetal. Halfmetallic materials are ferromagnets characterized by a $\downarrow$ subband which is completely filled, whereas the $\uparrow$ electrons provide metallic conductivity. Semimetals are reminiscent of ordinary paramagnetic semiconductors, except that they exhibit a negative 'energy gap'.

This work deals with NiMnSb layers covered by $\mathrm{Sb}$ overlayers. NiMnSb is a halfmetallic semi-Heusler alloy crystallizing in the cubic $\mathrm{Cl}_{\mathrm{b}}$ structure. It may be considered as a derivate of the parent Heusler alloy $\mathrm{Ni}_{2} \mathrm{MnSb}$ and has a $\downarrow$ band gap of less than about $0.5 \mathrm{eV}$ [1]. Antimony is a semimetal characterized by a very small negative energy gap [2]; the overlap and Fermi energies are about $180 \mathrm{meV}$ and $90 \mathrm{meV}$, respectively, and the electron and hole carrier densities are of comparable magnitude (about $5 \times 10^{-19} \mathrm{~cm}^{-3}$ ) [2]. The preparation and characterization of the sputtered NiMnSb films considered in this work has been described elsewhere [3-5]. The Sb grows epitaxially on NiMnSb, with a $<100\rangle$ orientation, a cubic structure, a $3.1 \AA$ lattice constant [6]. 
The magnetism of the $\mathrm{Sb}$ overlayer films was investigated by a combination of $\mathrm{X}$ ray absorption spectroscopy (XAS) and spin-polarized inverse photoemission spectroscopy (SPIPES) experiments [4,6]. Our approach yields a layer-specific analysis of the spin polarization, in contrast to methods such as that used in [7], where spin injection is probed indirectly, by considering the exchange coupling through a semiconducting medium.

The measured spin asymmetry exhibits an oscillatory behavior and extends quite well into the $\mathrm{Sb}$ layer (up to about $1 \mathrm{~nm}$ ). In the spin-dependent envelope-function approximation (SDEFA) used in [8], the unusual range of the spin polarization is explained by taking into account that there are no spin-down states available at the $\mathrm{NiMnSb}$ Fermi level. The $\uparrow$ electrons are able to move from the Sb overlayer into the $\mathrm{NiMnSb}$, whereas the $\downarrow$ electrons are reflected at the $\mathrm{NiMnSb} / \mathrm{Sb}$ interface. The latter boundary condition means that the wave functions $\psi_{\mathbf{k}} \downarrow(\mathbf{r})$ are equal to zero at $\mathrm{x}=0$. Using the solution of the spin-independent scattering at an infinite potential barrier [9] one obtains the $\downarrow$ electron density

$$
\mathrm{n}(\mathrm{x})=\frac{\mathrm{k}_{\mathrm{F}}^{3}}{3 \pi^{2}}\left(1+3 \frac{\cos \left(2 \mathrm{k}_{\mathrm{FX}}\right)}{\left(2 \mathrm{k}_{\mathrm{F}}\right)^{2}}-3 \frac{\sin \left(2 \mathrm{k}_{\mathrm{F}} \mathrm{x}\right)}{\left(2 \mathrm{k}_{\mathrm{F}}\right)^{3}}\right)
$$

which yields an oscilatory spin polarization $\mathrm{m}=(\mathrm{n} \uparrow-\mathrm{n} \downarrow) /(\mathrm{n} \uparrow+\mathrm{n} \downarrow)$ of the carrier electrons [8]. In other words, the comparatively long range of equilibrium spin injection from the half-metal NiMnSb into the $\mathrm{Sb}$ is explained by the semimetallic character of the $\mathrm{Sb}$ overlayer.

Here we start from a slightly different point of view. The NiMnSb/Sb interface is considered as a perturbation which creates a spin polarization in the Sb overlayer, and this perturbation is described in terms of a Landau-Ginzburg theory. This approach was originally designed to discuss strongly paramagnetic and weakly ferromagnetic dilute alloys [10], but it can also be used to describe thin-film problems $[11,12]$. In this paper, we discuss the electronic origin and the temperature dependence of the Landau-Ginzburg parameters and investigate the influence of boundary conditions.

\section{CALCULATION AND RESULTS}

\section{Gaussian Approximation}

Neglecting nonlinear contributions and restricting ourselves to long-wavelength terms (up to $\nabla^{2} \sim \mathrm{k}^{2}$ ) which we will discuss below, the energy functional can be written without considering higher-than-quadratic terms in k-space. The problem reduces to a LandauGinzburg equation:

$$
E=\int\left(\frac{C}{2}(\nabla m)^{2}+\frac{A}{2} m^{2}-h(\mathbf{r}) m\right) d \mathbf{r}
$$


In this equation, $\mathrm{m}(\mathbf{r})$ is the local magnetization, $\mathrm{C}$ can be interpreted as a kind of exchange stiffness, A indicates the tendency towards ferromagnetism, and $h(\mathbf{r})$ is an 'external' exchange field. In the present context, $h(\mathbf{r})$ is the exchange-field created by the $\mathrm{NiMnSb}$; in the $\mathrm{Sb}$ layer, $\mathrm{h}=0$.

Exploiting that the Gaussian approximation is exact for quadratic energy expressions we obtain the free energy:

$$
\mathrm{F}=\frac{1}{\mathrm{~V}_{\mathrm{e}}} \int\left(\frac{\mathrm{C}}{2}(\nabla \mathrm{m})^{2}+\frac{\mathrm{A}+\mathrm{k}_{\mathrm{B}} \mathrm{T}}{2} \mathrm{~m}^{2}-\mathrm{h}(\mathbf{r}) \mathrm{m}\right) \mathrm{d} \mathbf{r}
$$

where $V_{e}$ is the volume per electron. For $A<0$, this equation provides a very crude description of ferromagnetism below $T_{c}=|A| / k_{B}[13]$, whereas for paramagnets it yields a finite susceptibility at all temperatures.

Minimization of the free energy Eq. (3) yields

$$
-\mathrm{C} \nabla^{2} \mathrm{~m}+\left(\mathrm{A}+\mathrm{k}_{\mathrm{B}} \mathrm{T}\right) \mathrm{m}=\mathrm{h}(\mathbf{r})
$$

In the present case, $\mathrm{h}$ is the exchange field acting on the Sb spins at the interface. Since the three-dimensional character of the $\mathrm{Sb}$ electron gas is incorporated in the parameters $\mathrm{A}$ and $\mathrm{C}$, the resulting problem is one-dimensional, and the solutions of Eq. (4) are of the type $\exp ( \pm \mathrm{qx})$, where $\mathrm{q}=\left(\mathrm{A}+\mathrm{k}_{\mathrm{B}} \mathrm{T}\right)^{1 / 2} / \mathrm{C}^{1 / 2}$. In the simplest case, the spin polarization decays with an exponential decay length $\mathrm{R}=1 / \mathrm{q}$.

\section{Electronic origin of the Landau-Ginzburg terms}

Due to the small overlap of the conduction and valence bands the band-structure of the antimony conduction electrons can be treated as free-electron like. (True free-electron behavior is restricted to the $\Gamma$ point). The corresponding free-electron Fermi wave vector is of the order of $0.1 / \AA$, but due to the quite eccentric ellipsoidal shape of the Fermisurface pockets [2] this value is only semiquantitative.

The free-electron response to a weakly varying magnetic inhomogenity is described by $\mathrm{C}=1 / 12 \mathrm{D}\left(\mathrm{E}_{\mathrm{F}}\right) \mathrm{k}_{\mathrm{F}}^{2}, \mathrm{~A}=1 / \mathrm{D}\left(\mathrm{E}_{\mathrm{F}}\right)$, and $\mathrm{h}=\mu_{\mathrm{o}} \mu_{\mathrm{B}} \mathrm{H}(\mathbf{r})$ [14]. In a homogeneous field, where the C-term is unimportant, Eq. (4) reproduces the Pauli susceptibility $\chi_{\mathrm{p}}=$ $\mu_{\mathrm{o}} \mu_{\mathrm{B}} \mathrm{M}_{\mathrm{s}} \mathrm{D}\left(\mathrm{E}_{\mathrm{F}}\right)$. Coulomb interactions modify the the free-electron behavior, and for $\mathrm{A}=$ $1 / \mathrm{D}\left(\mathrm{E}_{\mathrm{F}}\right)-\mathrm{I}<0$, where $\mathrm{I}$ is the Stoner interaction parameter, Eq. (4) predicts ferromagnetism $[14,15]$. However, in antimony the density of states is very low, so that the spin susceptibility is very weak cannot compete against the diamagnetic contribution.

The free-electron parameters yield the zero-temperature decay length $(12)^{-1 / 2} / \mathrm{k}_{\mathrm{F}}$. The involvement of large wave vectors, caused by the sharp interface and seen as Friedel (or RKKY) oscillations, leads to somewhat larger effective decay lengths. Figure 1 compares the experimental data with theoretical predictions. Figure 1(a) shows the spin asymmetry at $\mathrm{E}_{\mathrm{F}}$ as a function of the Sb layer thickness. Only the $\mathrm{Sb}$ top layer is probed by this method, and the measured spin asymmetry characterizes, in crude way, the magnetic polarization of that layer. The lengths in Fig. 1(a) are given in arbitrary units, because it was not possible to obtain an exact value for the total coverlayer thickness 
(roughly, the range shown correspond to $1 \mathrm{~nm}$ ). Figure 1(b) shows a SDEFA prediction (solid line) with a corresponding Landau-Ginzburg decay (dashed line, $\mathrm{R}=0.941 / \mathrm{k}_{\mathrm{F}}$ ).

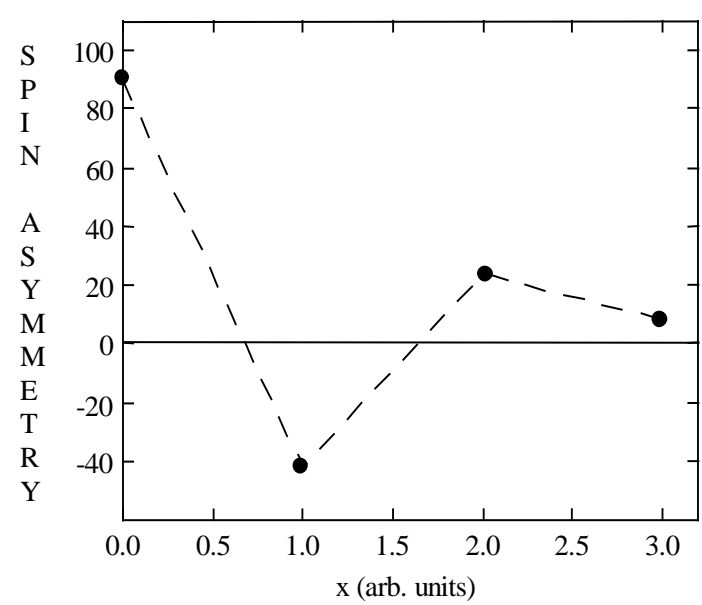

(a)

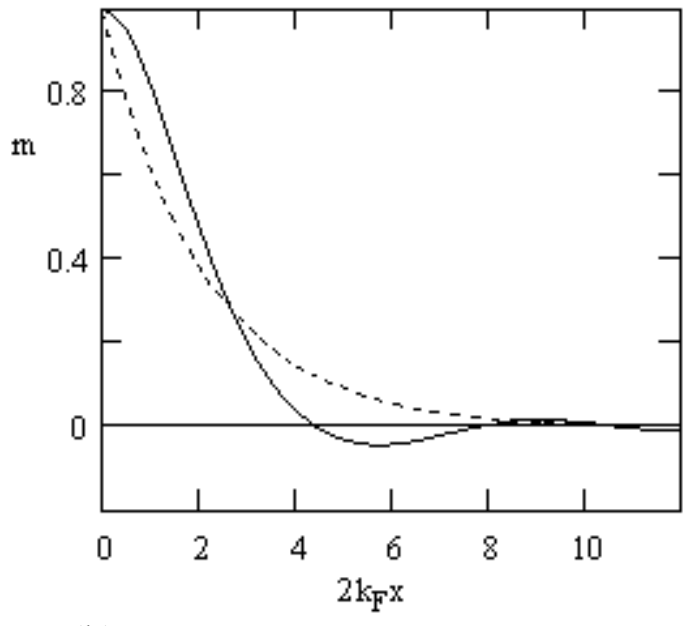

(b)

Fig. 1. Spin polarization in an NiMnSb: (a) spin-polarized inverse photoemission data at $\mathrm{E}_{\mathrm{F}}$ (the spin asymmetry is measured in \%) and (b) SDEFA and Landau-Ginzburg predictions.

\section{Temperature dependence}

Thermal activation leads to the involvement of excited electron states with small wavelengths. This effect tends to reduce the decay length. Neglecting the small exchange enhancement (Stoner parameter), we obtain

$$
R=\sqrt{\frac{C}{A+k_{B} T}}
$$

In the high-temperature limit, this amounts to a $1 / \sqrt{\mathrm{T}}$ law. On the other, $\mathrm{k}_{\mathrm{F}}$ and $\mathrm{D}\left(\mathrm{E}_{\mathrm{F}}\right)$ exhibit an intrinsic temperature dependence, associated for example with the thermal expansion of the lattice and the corresponding change of the overlap, so that $\mathrm{A}$ and $\mathrm{C}$ are temperature dependent quantities. This contribution is not necessarily much smaller than the explicit dependence shown in Eq. (5). Note that $\mathrm{R}$ can be interpreted as an equilibrium spin-penetration depth or equilibrium spin-injection length; it is not related to ballistic effects.

\section{$\underline{\text { Boundary conditions }}$}

For planar geometries, Eq. (4) yields solutions of the type $\exp ( \pm q x)$. The admixture of $\exp (q x)$ character depends on the boundary conditions. For semi-infinte $\mathrm{Sb}$,

$$
\mathrm{m}=\mathrm{m}_{\mathrm{o}} \exp (-\mathrm{qx})
$$


whereas for a thin film of thickness $t$

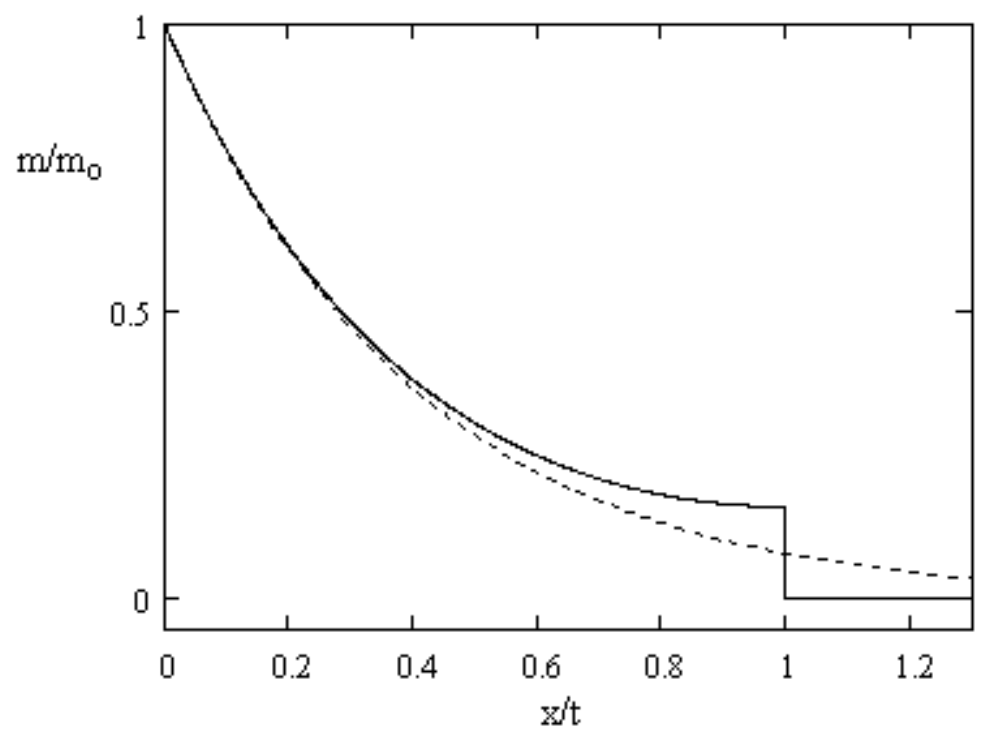

Fig. 2. Spin polarization in NiMnSb/Sb: Sb film of thickness $t$ (solid line) and seminfinite $\mathrm{Sb}$ (dashed line).

$$
m=m_{0} \frac{\cosh (q t-q x)}{\cosh (q t)}
$$

Figure 2 shows the difference between the two boundary conditions.

\section{DISCUSSION AND CONCLUSIONS}

The main difference between the SDEFA and Landau-Ginzburg approximations is the involvement of the Fermi surface. The SDEFA result (solid line in Fig. 1(b)) is of the RKKY type and exhibits oscillations arising from the sharp Fermi surface. As in the original RKKY theory [14], the oscillations mean that the electrons' finite wave vectors make it impossible to match perturbations on a local scale. However, our geometry differs from that of the original RKKY theory: we consider a planar geometry rather than the interaction between two localized moments. Note, furthermore, that our approach is reminiscent of the description of quantum confinement in thin-film semiconductors and loosely related to the approach by Hunziker and Landolt [7], where spin effects in semiconductors are discussed by considering the Heisenberg exchange between two hydrogen-like orbitals characterized by a small effective mass $\mathrm{m}^{*}$. (The effective mass is very low in semiconductors and semimetals.)

In the RKKY and SDEFA theories, the smearing of the Fermi surface due to thermal excitations suppresses the oscillatory character of the response and reduces the 
penetration length (decay length). Simply speaking, excited electron states have shorter wavelengths and their integral response to surfaces and impurities exhibits a higher resolution. By contrast, the Landau-Ginzburg theory is insensitive to details of the Fermi surface. The reason is that the Landau-Ginzburg energy is restricted to terms quadratic in $\mathrm{k}$ : there is no sharp wave-vector cutoff, and $\mathrm{k}_{\mathrm{F}}$ enters the energy only indirectly, by determining the parameters $\mathrm{A}$ and $\mathrm{C}$.

In conclusion, we have investigated the problem of equilibrium spin injection from the half-metal $\mathrm{NiMnSb}$ into the semi-metal $\mathrm{Sb}$. The comparatively long range of the $\mathrm{Sb}$ spin polarization is explained by the semimetallic character of the Sb overlayer, which leads to small Fermi wave vectors (wave-vector differences) $k_{F}$ and fairly large decay lengths. The decay is described in terms of a Landau-Ginzburg approach whose parameters are both electronic-structure and temperature dependent.

\section{ACKNOWLEDGMENT}

This work was supported by Office of Naval Research and the Nebraska Research Iniative.

\section{REFERENCES}

[1] R. A. de Groot and K. H. J. Buschow, J. Magn. Magn. Mater. 54-57 (1986) 1377.

[2] Y. Liu and R. E. Allen, Phys. Rev. B 52 (1995) 1566.

[3] C. N. Borca, T. Komesu, H.-K. Jeong, P. A. Dowben, D. Ristoiu, Ch. Hordequin, J. Pierre, and J. P. Nozières, Appl. Phys. Lett. 77 (2000) 88.

[4] D. Ristoiu, J. P. Nozières, C. N. Borca, B. Borca, P. A. Dowben, Appl. Phys. Lett. 76 (2000) 2349.

[5] D. Ristoiu, J. P. Nozieres, C. N. Borca, T. Komesu, H.-K. Jeong, and P. A. Dowben, Europhys. Lett. 43 (2000) 624.

[6] T. Komesu, C. N. Borca, H.-K. Jeong, P. A. Dowben, D. Ristoiu, J. P. Nozières, Sh. Stadler, Y. U. Idzerda, Phys. Lett. A 273 (2000) 245.

[7] M. Hunziker and M. Landolt, Phys. Rev. Lett. 84 (2000) 4713.

[8] R. Skomski, T. Komesu, C. N. Borca, H.-K. Jeong, P. A. Dowben, D. Ristoiu, and J. P. Nozières, J. Appl. Phys. (2001) in press.

[9] García-Moliner and F. Flores, "Introduction to the Theory of Solid Surfaces", University Press, Cambridge 1979.

[10] D. M. Edwards, J. Mathon, and E. P. Wohlfarth, J. Phys. F 5 (1975) 1619.

[11] J. Mathon, J. Phys. F 16 (1986) L217.

[12] P. A. Dowben, W. Hürsch, and M. Landolt, J. Magn. Magn. Mater. 125 (1993) 120-124.

[13] Q.-N. Qi, R. Skomski, and J. M. D. Coey, J. Phys. CM 6 (1994) 3245.

[14] W. Jones and N. H. March, "Theoretical Solid State Physics I", Wiley, London 1973.

[15] R. Skomski and J. M. D. Coey, "Permanent Magnetism", Institute of Physics, Bristol 1999. 